\title{
Micromechanical modeling of architected piezoelectric foam with simplified boundary conditions for hydrophone applications
}

\author{
Kamran A. Khan ${ }^{*}$, Hamad K. Alarafati ${ }^{2}$, Muhammad Ali Khan ${ }^{3}$ \\ ${ }^{1}$ Department of Aerospace Engineering, Khalifa University of Science and Technology, Abu Dhabi, \\ UAE \\ ${ }^{2}$ Department of Mechanical Engineering, Khalifa University of Science and Technology, Abu Dhabi, \\ UAE \\ ${ }^{3}$ School of Aerospace, Transport and Manufacturing, Cranfield University, Cranfield, UK
}

\begin{abstract}
Architected piezoelectric materials with controlled porosity are of interest for applications such as hydrophones, miniature accelerometers, vibratory sensors, and contact microphones. Current analytical modeling approach cannot be readily applied to design architected periodic piezoelectric foams with tunable properties while exhibiting elastic anisotropy and piezoelectric activity. This study presents micromechanical-finite element (FE) models to characterize the electromechanical properties of architected piezoelectric foams. The microstructure with zero-dimension (3-0 foam, spherical porosity) and one-dimensional (3-1 foam, cylindrical porosity) connectivity were considered to analyze the effect of porosity connectivity on the performance of piezoelectric foam. 3D FE models of the 3-0 and 3-1 foams were developed and using the intrinsic symmetry of porous structures simplified mixed boundary conditions (MBCs) equivalent to periodic boundary conditions (PBC) were proposed. The proposed approach is simple and eliminates the need of tedious mesh generation process on opposite boundary faces on the micromechanical model of porous microstructures with PBCs. The results obtained from the proposed micromechanics-FE models were compared with those obtained by means of the analytical models based on
\end{abstract}

\footnotetext{
${ }^{*}$ Corresponding author. Tel.: +971-(02) 4018227; Fax: +971-(02) 4472442

E-mail address: kamran.khan@ku.ac.ae (K.A. Khan)
} 
micromechanics theories, and FE models with PBCs reported in the literature for both 3-0 and 31 type foams. An excellent agreement was observed. The computed effective elastic, piezoelectric and dielectric properties and corresponding figure of merit (FOM) revealed that piezoelectric foams with 3-0 connectivity exhibit enhanced hydrostatic FOM as compared to piezoelectric foams with 3-1 connectivity. It is concluded that spherical porosity is more suitable to hydrophone applications.

Keywords: finite element analysis; electromechanical properties, architected porous materials, unit cell method, piezoelectric materials.

\section{Introduction}

Architected piezoelectric composites and foams owing to their unique electromechanical properties are of interest in wide range of actuators, sensors, medical devices, hydrophones and echo-cardiogram (Marselli et al., 1999). Piezoelectric composite have been produced by adding one or more constituents (Topolov and Bowen, 2008) while piezoelectric foam are produced by introducing controlled porosity (Hikita et al., 1983) in bulk piezoelectric materials. Piezoelectric foams with architected porosity can enhance signal-to-noise ratio, lower acoustic impedance, enhanced piezoelectric charge, voltage coefficients and hydrostatic figure of merit (FOM) (Khan et al., 2019). For example, piezoelectric foams showed higher hydrostatic FOM as compared to monolithic PZT for a similar hydrophone design (Geis et al., 2000). For various industrial and structural applications there is a need to explore various design of piezoelectric foam with tunable electromechanical properties through numerical experimentations.

The design of the piezoelectric foams are highly dependent on the spatial distribution of the porosity or its connectivity in a different direction (Newnham et al., 1978). Several experimental studies were conducted to understand the role of connectivity of porosity on the electromechanical 
properties of piezoelectric foams, such as, enclosed porosity (i.e.,3-0 foam) (Haun and Newnham, 1986; Kara et al., 2003; Ueda et al., 2010) cylindrical porosity (i.e.,3-1 foam) (Bast and Wersing, 1989); and open-foam like porosity (i.e.,3-3 type foam) (Lee et al., 2007; Nagata et al., 1980). These experiments conclude that porosity enhances the piezoelectric sensitivity of the piezoelectric foams.

Various analytical models were developed to predict the electromechanical properties of porous piezoelectric materials by considering simplified porosity configurations (3-0 and 3-1 types) (Banno, 1985), (Martin L. Dunn and Taya, 1993), (Mikata, 2001), (Bowen and Topolov, 2003). Current analytical models cannot be readily applied to compute electromechanical characteristics of architected periodic piezoelectric foams and composites exhibiting elastic anisotropy and piezoelectric activity.

Several researchers employed topology optimization techniques and the homogenization method to improve the performance of piezoelectric materials by designing new topologies of unit cells. Silva, Kikuchi, and co-workers (Silva et al., 1997), (Silva et al., 1998) proposed a topology optimization technique of finding the distribution of material and voids phases in a periodic unit cell that can optimize electromechanical efficiency of architected piezoelectric materials. Sigmund et al (Sigmund et al., 1998) employed the topology optimization method to design architected piezoelectric materials with optimal performance characteristics for hydrophone applications and their design proposed optimal three-dimensional anisotropic porous matrix microstructure.

Several micromechanical models based on finite element analysis were developed for predicting electromechanical properties of 3-0, 3-1 and 3-3 type porous piezoelectric materials and hence addressed more complex microstructures (Kar-Gupta and Venkatesh, 2008). These studies concluded that electromechanical properties of porous piezoelectric materials depend on level of 
porosity, pore shape, orientation and distribution, cellular interconnectivity and poling direction. Piezoelectric metamaterials and piezoelectric architected materials (e.g., (Iyer et al., 2015)) are the potential subclasses of porous piezoelectric materials. It is because their microstructure in any porosity configuration (such as 3-0,3-1 and 3-3) can be tailored to produce new innovative material designs with optimum multifunctionality required for specific applications (Wadley, 2006).

Currently, the designing of the piezoelectric foams and composite are performed using the unit cell (UC) homogenization method using periodic boundary conditions (PBCs) (Kar-Gupta and Venkatesh, 2008), (Iyer and Venkatesh, 2010). The application of PBCs requires UC periodicity and continuity of the displacements and the electrical potential, i.e., the difference of the displacements and electric potential for the corresponding points on the two opposite boundary surfaces should be specified. In this work, the symmetry of the periodic microstructure was recognized and an explicit mixed traction-displacement boundary conditions (BCs) for UCs were proposed while incorporating the continuity of electric electrical charge and displacement field to obtain the effective electromechanical properties of architected periodic piezoelectric foam and composites. The proposed approach is simple as there is no need to create a similar mesh on opposite faces of the unit cell, which require special consideration and added extra complexity in the modeling procedure of some complex architected porous microstructures. This study presents a micromechanical modeling and finite-element based computational homogenization framework to compute the electromechanical properties of architected periodic piezoelectric foam. As an example, 3D finite element models are developed to characterize the effects of porosity and constituents' material anisotropy on the electromechanical properties of 3-0 and 3-1 type piezoelectric foam. 


\section{Architecture of Porous Piezoelectric Materials}

We consider two typical periodic architected microstructures i.e., a 3-0 and 3-1 type piezoelectric foams with zero-dimension and one-dimensional connectivity, respectively. In 3-0 type and 3-1 type foams, the spherical and cylindrical porosity is embedded in an active piezoelectric matrix material, respectively. Figures 1 (a) and (b) show the architecture of 3-0 and 3-1 type piezoelectric foams with the direction of the poling axis. The piezoelectric matrix is made of piezoelectric system of $\mathrm{BaTiO}_{3}$ (poled in 3-direction). We have considered BaTiO3 in this study because there has been considerable interest in the community to explore the porous $\mathrm{BaTiO} 3$ in hydrophone (Iyer and Venkatesh, 2011) and energy harvesting applications (Roscow et al., 2015, 2017, 2018).

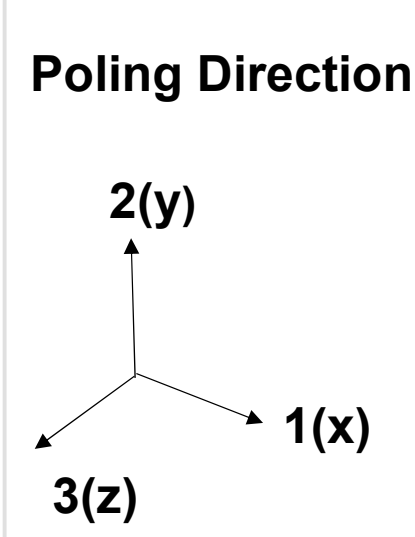

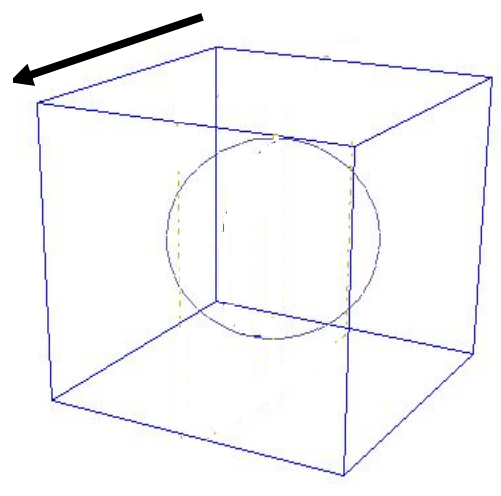

(a)

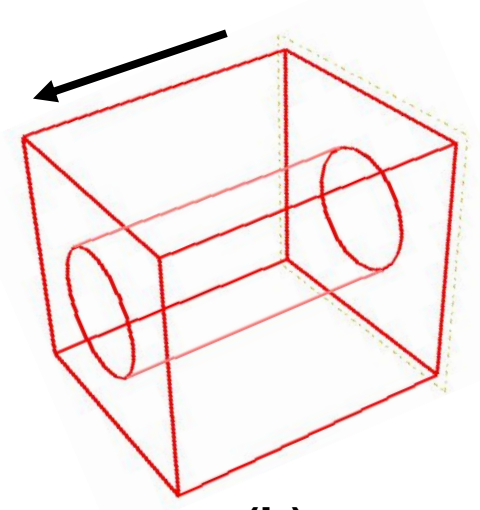

(b)

Figure 1. Architecture of Porous piezoelectric materials a) Spherical Porosity b) Cylindrical Porosity.

From a practical point of view, uniform poling can be possible in 3-1 type PMs (Getman and Lopatin, 1996) but poling 3-0 type PMs in a uniform manner could be a challenging task. It is anticipated that due to the presence of complex pore shape during the processing of poling the applied electric field concentrates in the low-permittivity pores region, which leads to an 
inhomogeneous electric field distribution throughout the cellular structure (Padurariu et al., 2012). Thus, some regions may remain un-poled or be poled in a direction that is different from the direction that is originally intended in the poling process. Recently, Zhang et al. (Zhang et al., 2018) examined the influence of pore volume fraction and morphology on the polarization-electric field behaviour of ferroelectric materials. It has been shown that the introduction of a low permittivity pore in a high-permittivity ceramic matrix lead to a broadening of the electric field distribution in the ceramic component and a decrease in the electric field experienced by the ceramic due to a concentration of the electric field in the lower permittivity pore region.

\section{Constitutive Model of Piezoelectric Material and Architected Foam}

The constitutive equations of monolithic piezoelectric material are assumed to be linear and can be written in compacted matrix strain-charge form as (Yang, 2006):

$$
\left\{\begin{array}{c}
\{\varepsilon\} \\
\{D\}
\end{array}\right\}=\left[\begin{array}{cc}
{[S]^{E}} & {[d]} \\
{[d]^{T}} & {[\mathbf{\kappa}]^{\sigma}}
\end{array}\right]\left\{\begin{array}{l}
\{\sigma\} \\
\{E\}
\end{array}\right\}
$$

Where $\{\varepsilon\},\{\sigma\},\{D\}$, and $\{E\}$ are the field variables, and denote the strain tensor, stress tensor, electric displacement vector, and electric field vector, respectively. The material constants $\left[S^{E}\right]$, $\left[\kappa^{\sigma}\right]$, and $[d]$ denotes the compliance tensor, dielectric tensor and the piezoelectric strain tensor, respectively. The superscripts $E$ and $\sigma$ indicate that the values of materials constants are measured at zero or constant electric field and zero or constant stress, respectively. The components of stress and strain tensor can be written in Voight notation, as $\sigma=\left\{\sigma_{1}, \sigma_{2}, \sigma_{3}, \sigma_{4}, \sigma_{5}, \sigma_{6}\right\}^{T}$ and $\varepsilon=$ $\left\{\varepsilon_{1}, \varepsilon_{2}, \varepsilon_{3}, \varepsilon_{4}, \varepsilon, \varepsilon_{6}\right\}^{T}$. The superscript ' $\mathrm{T}$ ' denotes the transpose. The third order piezoelectric strain tensor and fourth order compliance tensor can be reduced in matrix form as $d_{i k l}=d_{i b}$ and $S_{i j k l}=$ $S_{a b}$, respectively. Further discussion regarding the piezoelectric material notations and its behavior 
can be found in (Yang, 2006). For transversely isotropic piezoelectric solid, the compliance, the piezoelectric, and the dielectric matrices involves 11 independent coefficients. The constitutive equation can be written in expanded matrix form as (Berger et al., 2006):

$$
\left(\begin{array}{c}
\varepsilon_{1} \\
\varepsilon_{2} \\
\varepsilon_{3} \\
\varepsilon_{4} \\
\varepsilon_{5} \\
\varepsilon_{6} \\
D_{1} \\
D_{2} \\
D_{3}
\end{array}\right)=\left[\begin{array}{ccccccccc}
S_{11}^{E} & S^{E}{ }_{12} & S^{E}{ }_{13} & 0 & 0 & 0 & 0 & 0 & -d_{31} \\
S^{E}{ }_{12} & S^{E}{ }_{22} & S^{E}{ }_{23} & 0 & 0 & 0 & 0 & 0 & -d_{32} \\
S^{E}{ }_{13} & S^{E}{ }_{23} & S^{E}{ }_{33} & 0 & 0 & 0 & 0 & 0 & -d_{33} \\
0 & 0 & 0 & S^{E}{ }_{44} & 0 & 0 & 0 & -d_{24} & 0 \\
0 & 0 & 0 & 0 & S^{E}{ }_{55} & 0 & -d_{15} & 0 & 0 \\
0 & 0 & 0 & 0 & 0 & S^{E} & 0 & 0 & 0 \\
0 & 0 & 0 & 0 & d_{15} & 0 & \boldsymbol{\kappa}_{11}^{\sigma} & 0 & 0 \\
0 & 0 & 0 & d_{24} & 0 & 0 & 0 & \boldsymbol{\kappa}_{22}^{\sigma} & 0 \\
d_{31} & d_{32} & d_{33} & 0 & 0 & 0 & 0 & 0 & \boldsymbol{\kappa}_{33}^{\sigma}
\end{array}\right)\left(\begin{array}{l}
\sigma_{1} \\
\sigma_{2} \\
\sigma_{3} \\
\sigma_{4} \\
\sigma_{5} \\
\sigma_{6} \\
E_{1} \\
E_{2} \\
E_{3}
\end{array}\right)
$$

The Eq. (1) can be used to represent the effective response of the architected piezoelectric foam by considering the volume average of the piezoelectric field variables, which can be written in compacted matrix form (Martínez-Ayuso et al., 2017), i.e.,

$$
\left\{\begin{array}{c}
\{\bar{\varepsilon}\} \\
\{\bar{D}\}
\end{array}\right\}=\left[\begin{array}{ll}
{[\bar{S}]^{E}} & {[\bar{d}]} \\
{[\bar{d}]^{T}} & {[\overline{\mathbf{\kappa}}]^{\sigma}}
\end{array}\right]\left\{\left\{\begin{array}{l}
\bar{\sigma}\} \\
\bar{E}\}
\end{array}\right\}\right.
$$

where, $\{\bar{\varepsilon}\},\{\bar{\sigma}\},\{\bar{D}\}$, and $\{\bar{E}\}$ are the effective strains, stresses, electric displacement, and electric field. Based on Eq. (3), the complete characterization of the effective electromechanical properties of architected piezoelectric foam requires quantifying 45 independent material constants, i.e., 21 effective elastic compliance, 18 effective piezoelectric and 6 effective dielectric constants.

\section{Analytical Model}

The micromechanical modeling approach was used by Dunn and Taya (Martin L. Dunn and Taya, 1993; M. L. Dunn and Taya, 1993) to determine the effective electromechanical properties of a two-phase piezoelectric composite and porous piezoelectric materials as a function of volume fraction/porosity and typical microstructures of secondary phase including continuous cylinder, 
ribbon-like, and ellipsoidal. Next, we briefly describe the theoretical approach used to model the effective electromechanical behavior of porous piezoelectric materials. The piezoelectric materials constitutive relations described in $\mathrm{Eq}$ (1) can be represented as

$\Sigma_{i J}=E_{i J M n} Z_{M n}$

Where $Z_{M n}, \Sigma_{i j}$, and $\mathrm{E}_{i J M n}$ are not tensors and $\mathrm{E}_{i J M n}$ represents the electro-elastic moduli matrix. where lowercase subscripts take on the range 1,2,3 while upper case subscripts take on the range $1,2,3,4$. In terms of the field variables discussed in Eq (1),

$Z_{M n}=\left\{\begin{array}{cr}\varepsilon_{m n}, & (M=1,2,3) \\ -\phi_{, n}, & (M=4)\end{array}\right.$

$\Sigma_{i J}=\left\{\begin{array}{lr}\sigma_{i j}, & (J=1,2,3) \\ D_{i}, & (J=4)\end{array}\right.$

$\mathrm{E}_{i J M n}=\left\{\begin{array}{cr}C_{i j m n}, & (J, M=1,2,3) \\ e_{n i j}, & (J=1,2,3 ; M=4) \\ \sigma_{i m n}, & (J=4 ; M=1,2,3) \\ \kappa_{i n}, & (J, M=4)\end{array}\right.$

where $\sigma_{i j}, \varepsilon_{i j}, D_{i}$, and $\phi_{, i}$ are the stress, strain, electric displacement, and electric potential gradient, respectively. The effective behavior of two-phase perfectly bonded piezoelectric composites can be related by using the volume average of the piezoelectric field variables $\left(\sigma_{i j}, \varepsilon_{i j}, D_{i}\right.$, and $\left.\phi_{, i}\right)$

$\overline{\boldsymbol{\Sigma}}=\boldsymbol{E} \overline{\mathbf{Z}}$

$\overline{\boldsymbol{\Sigma}}=(1-c) \overline{\boldsymbol{\Sigma}}_{\mathbf{1}}+\boldsymbol{c} \overline{\boldsymbol{\Sigma}}_{\mathbf{2}}$

$\overline{\mathbf{Z}}=(1-c) \overline{\mathbf{Z}}_{\mathbf{1}}+c \overline{\mathbf{Z}}_{\mathbf{2}}$

Where the overbar represents the volume average of a quantity and bold letter denote matrix $(9 \mathrm{x}$ 1 or $9 \times 9$ ) quantities. The $c$ denotes the volume fraction of the porous phase and subscript 1 and 2 denote the two piezoelectric phases, where 1 represent the matrix phase. Using the constitutive relation of each phase and Eq. (8)- (10), the effective electro-elastic moduli can be represented as 
$\mathbf{E}=\mathbf{E}_{\mathbf{1}}+(1-c)\left(\mathbf{E}_{\mathbf{2}}-\mathbf{E}_{\mathbf{1}}\right) \boldsymbol{A}$

Where $\mathrm{A}$ is the strain-potential gradient concentration matrix which related the average strain and potential gradient in phase 2 to that in the composite, which can be defined according to MoriTanaka (Mori and Tanaka, 1973) effective medium theory as

$\mathbf{A}=\left[I+(1-c) S E_{1}^{-1}\left(\mathbf{E}_{2}-\mathbf{E}_{1}\right)\right]^{-1}$

where $\mathrm{S}$ is the constraint tensor and is a function of only the shape of the inclusion and the electroelastic moduli of the matrix. For porous materials $E_{2}=0$ and the effective electro-elastic moduli reduce to

$\mathbf{E}=\mathbf{E}_{\mathbf{1}}\left\{\boldsymbol{I}-c[\boldsymbol{I}-(1-c) \boldsymbol{S}]^{-\mathbf{1}}\right\}$

The expression of effective electro-elastic moduli depends on $\mathrm{E}_{1}, \mathrm{c}$ and shape of the architecture shape through the constraint tensor $\mathrm{S}$. The $\mathrm{S}$ is the coupled electro-elastic tensor analogous to Eshelby's tensor and can be expressed in terms of surface integrals over the unit sphere (M. L. Dunn and Taya, 1993) and its components are determined explicitly for 3-0 type porosity (ellipsoidal inclusions (Dunn, 1994), spherical inclusion (Mikata, 2001)), and for 3-1 type porosity (Martin L. Dunn and Taya, 1993) in a transversely isotropic matrix. For 3-1 type porosity, the nonzero components of the piezoelectric Eshelby's tensor S are explicitly obtained as

$$
\begin{aligned}
& \mathrm{S}_{1111}=\mathrm{S}_{1111}=\left(5 C_{11}+C_{12}\right) /\left(8 C_{11}\right) \\
& \mathrm{S}_{1212}=\mathrm{S}_{2121}=\mathrm{S}_{1221}=\mathrm{S}_{2112}=\left(3 C_{11}-C_{12}\right) /\left(8 C_{11}\right) \\
& \mathrm{S}_{1313}=\mathrm{S}_{3131}=\mathrm{S}_{1331}=\mathrm{S}_{3113}=\mathrm{S}_{2323}=\mathrm{S}_{3232}=\mathrm{S}_{2332}=\mathrm{S}_{3223}=1 / 4 \\
& \mathrm{~S}_{1122}=\mathrm{S}_{2211}=\left(3 C_{12}-C_{11}\right) /\left(8 C_{11}\right) \\
& \mathrm{S}_{1133}=\mathrm{S}_{2233}=\left(C_{13}\right) /\left(2 C_{11}\right) \\
& \mathrm{S}_{1143}=\mathrm{S}_{2243}=\left(e_{31}\right) /\left(2 C_{11}\right)
\end{aligned}
$$


$\mathrm{S}_{4141}=\mathrm{S}_{4242}=1 / 2$

For 3-0 type spherical porosity, the explicit expressions for the non-zero components of piezoelectric Eshelby's tensor S were determined by Mikata (Mikata, 2001). There were 38 nonzero components of constraint tensor, so for brevity we are not presenting the explicit expressions.

\section{Micromechanical Modeling of Architected Porous Piezoelectric Materials}

In this study, we have extended our previously developed micromechanical-FE homogenization framework (Khan and Abu Al-Rub, 2017) for porous elastic solids to periodic architected piezoelectric materials. A mixed boundary conditions equivalent to PBC are developed and applied on a unit cell (UC) to fully characterize the effective electromechanical properties of periodic architected piezoelectric materials based upon the properties of base materials and topological configuration in the UC.

Finite element models of 3-0 and 3-1 type piezoelectric foam are generated by varying the porosity values ranging from $0-50 \%$ with spherical and cylindrical porosity embedded in an active piezoelectric matrix material, respectively. Figure 2 (a) and (b) show a representative UC of both 3-0 and 3-1 type piezoelectric foams, which are assumed to be poled longitudinally (poled along the z-axis) and to be made of Barium Titanate (BaTiO3). The commercial finite element analysis software ABAQUS@ was used to carry out the analysis and assumed that all regions in the UC of piezoelectric foam are uniformly poled in a specific direction. The electromechanical properties of BaTiO3 are given in Table 1 (Kar-Gupta and Venkatesh, 2006). Figure 2 also shows the example of the UC showing 6 boundary faces with respect to the axes-directions. Each UC has meshed with 10-noded quadratic piezoelectric tetrahedron elements (C3D10E). Each node in the FE model has

a total four degree of freedom, three displacements $\left(u_{1}, u_{2}, u_{3}\right)$ and one electrical potential $(\phi)$. To 
avoid rigid body motion under electrical loading, the location of arbitrary points $\mathrm{A}, \mathrm{B}$, and $\mathrm{C}$ are also shown that are constrained specifically that will be discussed later.
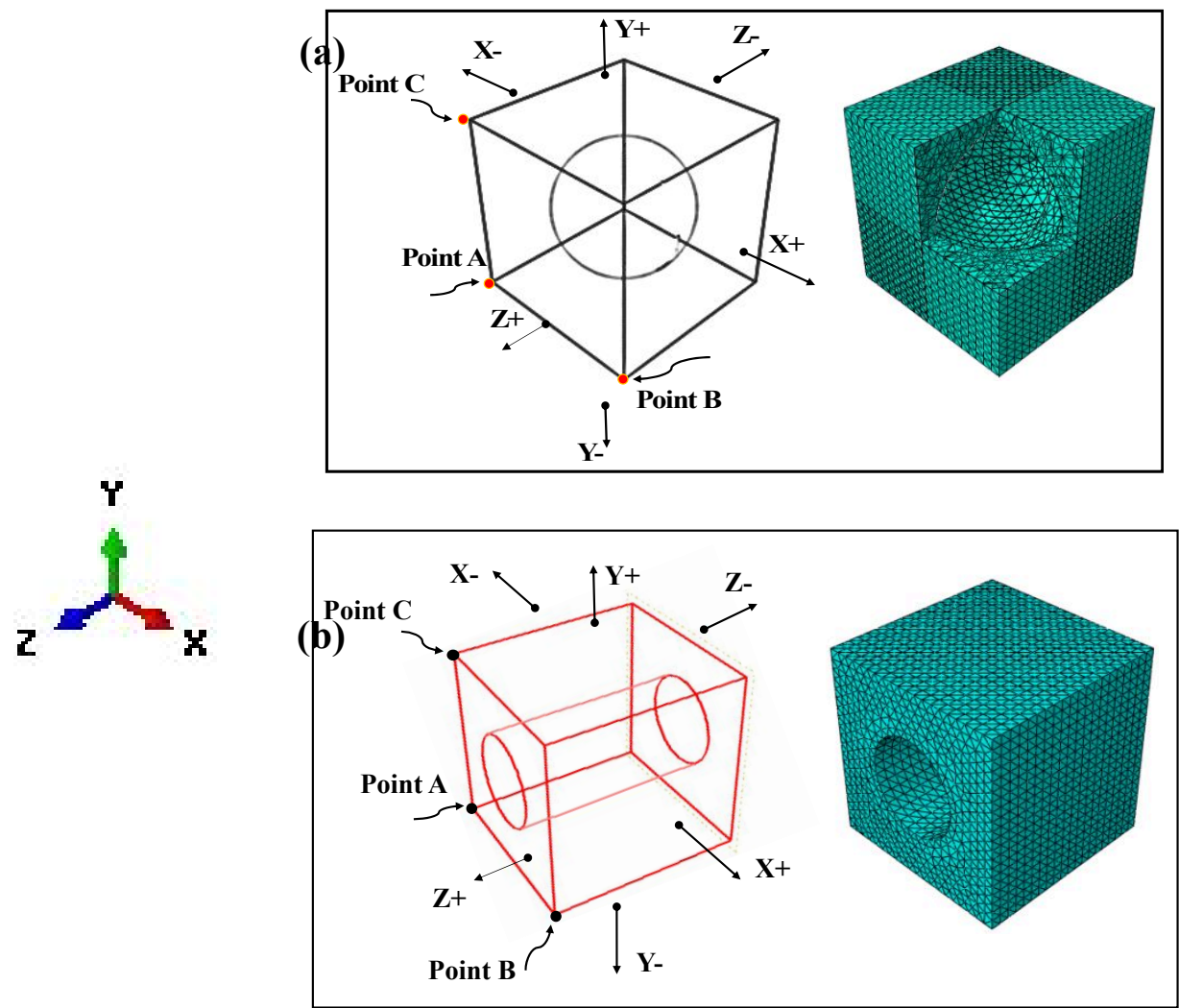

Figure 2. Example of a UC showing 6 boundary faces with respect to the axes directions and meshed UC with 10-noded quadratic piezo-electric tetrahedron elements (C3D10E). (a) spherical porosity, (b) cylindrical porosity

Table 1. Properties of the model piezoelectric system of $\mathrm{BaTiO}_{3}$ (poled in 3-direction)

\begin{tabular}{|l|l|}
\hline Properties & $\mathrm{BaTiO}_{3}$ \\
\hline$E_{1}=E_{3}(\mathrm{GPa})$ & 109.9 \\
$E_{3}(\mathrm{GPa})$ & 105.2 \\
$G_{13}=G_{23}(\mathrm{GPa})$ & 43.86 \\
$G_{12}(\mathrm{GPa})$ & 42.4 \\
$v_{12}=v_{21}$ & 0.297 \\
$v_{13}=v_{23}$ & 0.319 \\
$v_{31}=v_{32}(\mathrm{~m} / \mathrm{V})$ & 0.305 \\
$d_{15}=d_{24}(\mathrm{~m})$ & 260
\end{tabular}




\begin{tabular}{|l|l|}
\hline$d_{31}=d_{32}(\mathrm{p} \mathrm{m} / \mathrm{V})$ & -78 \\
$d_{33}(\mathrm{p} \mathrm{m} / \mathrm{V})$ & 190 \\
$\kappa_{11}^{\varepsilon}=\kappa_{22}^{\varepsilon}(\mathrm{n} \mathrm{C} / \mathrm{V} \mathrm{m})$ & 12.8 \\
$\kappa_{33}^{\varepsilon}(\mathrm{n} \mathrm{C} / \mathrm{V} \mathrm{m})$ & 15.1 \\
Permittivity of free space, $\kappa_{0}$ & $8.85 \times 10^{-12}$ \\
Density, $\rho\left(\mathrm{kg} / \mathrm{m}^{3}\right)$ & 5700 \\
\hline
\end{tabular}

Periodic boundary conditions (PBCs) are usually imposed on UC to determine the effective responses of infinitely repeating patterns of periodic architecture ((Kanit et al., 2003), (Khan and Muliana, 2009), (Choudhry et al., 2016)). PBCs yield acceptable results as compared to the ones obtained from homogeneous traction or displacement boundary conditions ((Jiang et al., 2002), (Xia et al., 2003)). Ensuring the continuity of displacements and the electrical potential and periodicity of UC, Xia et al. (Xia et al., 2003) derived PBCs in terms of normal average stretches and contractions $\left(c_{i}^{j}, i=j=1,2,3\right)$ of the UC and shear deformations $\left(c_{i}^{j}, i \neq j\right)$, i.e., $u_{i}^{j+}(\mathrm{x}, \mathrm{y}, \mathrm{z})-u_{i}^{j-}(\mathrm{x}, \mathrm{y}, \mathrm{z})=c_{i}^{j} \quad(i, j=1,2,3)$ where $j^{+}$means along the positive $X_{j}$ direction and $j^{-}$means along the negative $X_{j}$ direction. Similarly, the PBCs for the electric potential are given by $\phi^{j+}(\mathrm{x}, \mathrm{y}, \mathrm{z})-\phi^{j-}(\mathrm{x}, \mathrm{y}, \mathrm{z})=\overline{E_{i}}\left(\mathrm{x}_{i}^{j+}-x_{i}^{j-}\right) \quad(i=1,2,3) \quad$, where $\quad \bar{E}_{i} \quad$ is the applied macroscopic electric field.

The PBCs requires that the difference of the displacements for the corresponding points on the two opposite boundary surfaces should be specified, which requires sometime a tedious mesh 
generation process when architected periodic microstructures is complex. For periodic microstructures, $\mathrm{Li}(\mathrm{Li}, 2008)$ derived a set of mixed boundary conditions that is equivalent to PBCs to compute their elastic response. Recently, Khan and Khan (Khan and Khan, 2019) extended Li's (Li, 2008) work and incorporated continuity of electric electrical charge to derive the complete set of mixed B.Cs and obtained effective electromechanical properties of architected periodic piezoelectric foams, as given in Table 2. To compute effective electroelastic moduli, the volume average of the piezoelectric field variables is needed. Therefore, the average stress and strain were obtained by volume (V) integration over the UC, such that:

$\bar{\sigma}_{i j}=\frac{1}{V} \int_{V} \sigma_{i j}(x, y, z) d V \quad \bar{\varepsilon}_{i j}=\frac{1}{V} \int_{V} \varepsilon_{i j}(x, y, z) d V$

Analogously the average electric fields and electrical displacements are defined by

$\bar{E}_{i}=\frac{1}{V} \int_{V} E_{i}(x, y, z) d V \quad \bar{D}_{i}=\frac{1}{V} \int_{V} D_{i}(x, y, z) d V$

Using traction continuity one can find that the average stress is represented by

$\bar{\sigma}_{i j}=\frac{R_{i j}}{A_{j}}($ no summation on $\mathrm{j})$

Using electrical charge continuity, the average electric displacement is represented by

$$
\bar{D}_{i}=\frac{q_{i}}{A_{i}}
$$

Eq. (23) demonstrates that the average stress over the UC can be simply obtained by dividing the resultant tractions $\left(R_{i j}\right)$ on the boundary surfaces from the areas $\left(A_{j}\right)$ of the corresponding boundary surfaces. Eq. 8 demonstrates that the average electrical displacement over the UC can 
be simply obtained by dividing the resultant charge $\left(q_{i}\right)$ on the boundary surfaces from the areas ( $A_{i}$ ) of the corresponding boundary surfaces.

Table 2. Complete set of boundary conditions with computation formulae to determine electromechanical coefficients.

\begin{tabular}{|c|c|c|c|c|c|c|c|c|}
\hline $\begin{array}{l}\text { Load } \\
\text { Case }\end{array}$ & $\begin{array}{c}\text { Coefficie } \\
\text { nts }\end{array}$ & $\mathbf{X}^{-}$ & $\mathbf{X}^{+}$ & $\mathbf{Y}^{-}$ & $\mathbf{Y}^{+}$ & $\mathbf{Z}^{-}$ & $\mathbf{Z}^{+}$ & Relation \\
\hline & & $\bar{u}_{i} / \bar{\phi}$ & $\bar{u}_{i} / \bar{\phi}$ & $\bar{u}_{i} / \bar{\phi}$ & $\bar{u}_{i} / \bar{\phi}$ & $\bar{u}_{i} / \bar{\phi}$ & $\bar{u}_{i} / \bar{\phi}$ & \\
\hline 1 & $\begin{array}{l}\bar{S}_{11}^{E}, \bar{S}_{12}^{E} \\
\bar{S}_{13}^{E}, \bar{d}_{31}^{E}\end{array}$ & $0 / 0$ & $\overline{u_{1}} / 0$ & $0 / 0$ & $-/ 0$ & $0 / 0$ & $-/ 0$ & $\begin{array}{c}\bar{\varepsilon}_{1} / \bar{\sigma}_{1}, \bar{\varepsilon}_{2} / \bar{\sigma}_{1} \\
\bar{\varepsilon}_{3} / \bar{\sigma}_{1}, \bar{D}_{3} / \\
\bar{\sigma}_{1}\end{array}$ \\
\hline 2 & $\begin{array}{l}\bar{S}_{21}^{E}, \bar{S}_{22}^{E} \\
\bar{S}_{23}^{E}, \bar{d}_{32}^{E}\end{array}$ & $0 / 0$ & $-/ 0$ & $0 / 0$ & $u_{2} / 0$ & $0 / 0$ & $-/ 0$ & $\begin{array}{l}\bar{\varepsilon}_{1} / \bar{\sigma}_{2}, \bar{\varepsilon}_{2} / \bar{\sigma}_{2} \\
\bar{\varepsilon}_{3} / \bar{\sigma}_{2}, \bar{D}_{3} / \bar{\sigma}_{2}\end{array}$ \\
\hline 3 & $\begin{array}{l}\bar{S}_{31}^{E}, \bar{S}_{32}^{E} \\
\bar{S}_{33}^{E}, \bar{d}_{33}^{E}\end{array}$ & $0 / 0$ & $-/ 0$ & $0 / 0$ & $-/ 0$ & $0 / 0$ & $\overline{u_{3}} / 0$ & $\begin{array}{c}\bar{\varepsilon}_{1} / \bar{\sigma}_{3}, \bar{\varepsilon}_{2} / \bar{\sigma}_{3} \\
\bar{\varepsilon}_{3} / \bar{\sigma}_{3}, \bar{D}_{3} / \\
\bar{\sigma}_{3}\end{array}$ \\
\hline 4 & $\bar{S}_{44}^{E}, \bar{d}_{24}^{E}$ & $\begin{array}{c}\overline{u_{2}}=0 \\
\overline{u_{3}}=0 \\
/ 0\end{array}$ & $\begin{array}{c}\frac{u_{2}}{u_{3}}=0 \\
/ 0\end{array}$ & $\begin{array}{c}\overline{u_{1}}=0 \\
u_{3}=0 \\
/ 0\end{array}$ & $\begin{array}{c}\frac{u_{1}}{u_{3}}=0 \\
/ 0\end{array}$ & $\begin{array}{c}\overline{u_{3}}=0 \\
/ 0\end{array}$ & $\begin{array}{c}\overline{u_{3}}=0 \\
/ 0\end{array}$ & $\begin{array}{l}\bar{\varepsilon}_{4} / \bar{\sigma}_{4} \\
\bar{D}_{2} / \bar{\sigma}_{4}\end{array}$ \\
\hline 5 & $\bar{S}_{55}^{E}, \bar{d}_{15}^{E}$ & $\begin{array}{c}\overline{u_{2}}=0 \\
\overline{u_{3}}=0 \\
/ 0\end{array}$ & $\begin{array}{c}\overline{u_{2}}=0 \\
u_{3}=0 \\
/ 0\end{array}$ & $\begin{array}{c}u_{2}=0 \\
/ 0\end{array}$ & $\begin{array}{c}u_{2}=0 \\
/ 0\end{array}$ & $\begin{array}{c}\frac{u_{1}}{u_{2}}=0 \\
=0 \\
/ 0\end{array}$ & $\begin{array}{c}\frac{u_{1}}{u_{2}}=0 \\
/ 0\end{array}$ & $\begin{array}{l}\bar{\varepsilon}_{5} / \bar{\sigma}_{5} \\
\bar{D}_{1} / \bar{\sigma}_{5}\end{array}$ \\
\hline 6 & $\bar{S}_{66}^{E}$ & $\begin{array}{c}u_{1}=0 \\
/ 0\end{array}$ & $\begin{array}{c}\overline{u_{1}}=0 \\
/ 0\end{array}$ & $\begin{array}{c}\frac{u_{1}}{u_{3}}=0 \\
/ 0\end{array}$ & $\begin{array}{c}\frac{u_{1}}{u_{3}}=0 \\
/ 0\end{array}$ & $\begin{array}{c}\overline{u_{1}}=0 \\
\overline{u_{2}}=0 \\
/ 0\end{array}$ & $\begin{array}{c}\overline{u_{1}}=0 \\
\overline{u_{2}} \neq 0 \\
/ 0\end{array}$ & $\bar{\varepsilon}_{6} / \bar{\sigma}_{6}$ \\
\hline 7 & $\bar{d}_{15}^{\sigma}, \bar{\kappa}_{11}^{\sigma}$ & $* / 0$ & $-/ \bar{\phi}$ & $-1-$ & $-1-$ & $-1-$ & $-1-$ & $\begin{array}{l}\bar{\varepsilon}_{5} / \bar{E}_{1} \\
\bar{D}_{1} / \bar{E}_{1}\end{array}$ \\
\hline 8 & $\bar{d}_{24,}^{\sigma}, \bar{\kappa}_{22}^{\sigma}$ & $-1-$ & $-1-$ & $* / 0$ & $-/ \bar{\phi}$ & $-1-$ & $-1-$ & $\begin{array}{l}\bar{\varepsilon}_{4} / \bar{E}_{2} \\
\bar{D}_{2} / \bar{E}_{2}\end{array}$ \\
\hline 9 & $\begin{array}{l}\bar{d}_{31}^{\sigma}, \bar{d}_{32}^{\sigma} \\
\bar{d}_{33}^{\sigma}, \bar{\kappa}_{33}^{\sigma}\end{array}$ & $-1-$ & $-1-$ & $-1-$ & $-1-$ & $* / 0$ & $-/ \bar{\phi}$ & $\begin{array}{l}\bar{\varepsilon}_{1} / \bar{E}_{3}, \bar{\varepsilon}_{2} / \bar{E}_{3} \\
\bar{\varepsilon}_{3} / \bar{E}_{3}, \bar{D}_{3} / \bar{E}_{3}\end{array}$ \\
\hline
\end{tabular}

*Points $\mathrm{A}, \mathrm{B}$ and $\mathrm{C}$ are constrained on respective faces (having zero electric potential) to avoid rigid body motion.

\section{Results and Discussion}

To completely characterize the elastic, piezoelectric and dielectric properties of architected piezoelectric foam, the nine load-case are applied to the UCs as shown in Table 2. The BCs were 
applied such that for each load case there is only one non-zero component of effective stress and electric field vector remains on the right side of the Eq. (2). For each load case, the computation of some of the typical electromechanical coefficients are also shown in Table 2. Results of these loading cases can yield 45 independent constants required to completely characterize an architected piezoelectric foam. Once the compliance tensor $\left[\boldsymbol{S}^{E}\right]$, dielectric tensor $\left[\boldsymbol{\kappa}^{\sigma}\right]$, and the piezoelectric strain tensor $[\boldsymbol{d}]$ are determined, the following relations are used to obtain the other constants that can be used to evaluate the performance of the porous piezoelectric materials.

$$
\begin{aligned}
& {\left[\boldsymbol{C}^{E}\right]=\left[\boldsymbol{S}^{E}\right]^{-1},[\boldsymbol{e}]=[\boldsymbol{d}]\left[\boldsymbol{C}^{E}\right],\left[\boldsymbol{\kappa}^{\bar{\varepsilon}}\right]=\left[\boldsymbol{\kappa}^{\sigma}\right] € \boldsymbol{\phi}\left[\boldsymbol{e}^{T},\right.} \\
& {\left[\boldsymbol{S}^{D}\right]=\left[\boldsymbol{S}^{E}\right]-[\boldsymbol{d}]^{T}[\boldsymbol{g}],\left[\boldsymbol{C}^{D}\right]=\left[\boldsymbol{S}^{D}\right]^{-1}\left[\mathrm{~g} \in \boldsymbol{f} \boldsymbol{h}\left[\boldsymbol{S}^{D}\right], \boldsymbol{h}=\left[\boldsymbol{k}^{\boldsymbol{f}}\right]\right] \boldsymbol{e} .}
\end{aligned}
$$

where $\left[\boldsymbol{C}^{D}\right],\left[\boldsymbol{S}^{D}\right],[\boldsymbol{e}],\left[\boldsymbol{\kappa}^{\varepsilon}\right],[\boldsymbol{g}],[\boldsymbol{h}]$ are the stiffness tensor for zero electric displacement, compliance tensor for zero electric displacement, piezoelectric stress tensor, dielectric strain tensor, piezoelectric voltage constants, and piezoelectric current constants, respectively.

Next, we evaluated the electromechanical properties of the UC and compared our computed coefficients values with the ones reported in the literature. Here, we compared our results with Iyer and Venkatesh (2010) and Ronit and Venkatesh (2006), who studied a similar 3-0 and 3-1 type piezoelectric foam, respectively, but employed PBCs to compute the effective properties. Moreover, we also compared our results with the analytical solutions of Dunn and Taya (1993) and Mikata (2001) for 3-1 and 3-0 type piezoelectric foam, respectively. It is emphasized here that we have compared only those results which are available in the literature. For both 3-0 and 3-1 foams, we applied the load and boundary conditions as per nine loading cases shown in Table 2 to compute the components of $[\overline{\boldsymbol{S}}]^{E}$, $[\overline{\boldsymbol{d}}]$, and $[\overline{\boldsymbol{\kappa}}]^{\sigma}$ matrices and then using Eq. (25) evaluate the components of $[\overline{\boldsymbol{C}}]^{E}$ and $[\overline{\boldsymbol{e}}]$ matrices. 
For the calculation of the effective compliance matrix coefficients $\bar{S}_{11}^{E}, \bar{S}_{12}^{E}, \bar{S}_{13}^{E}$, and $\bar{d}_{31}^{E}$, the boundary conditions have to be applied to the UC in such a way that, except the stress in the direction-1 $\left(\bar{\sigma}_{1}\right)$, all other macroscopic stresses and gradients of electric potential $\left(\bar{E}_{i}\right)$ become zero. We prescribed a displacement in $\mathrm{x}$ direction on surface $\mathrm{X}+$ as shown in Figure 3 (a) and (b). The overall strain is computed by dividing the prescribed displacement from the length of the UC along the direction-1. For the calculation of the average stress $\bar{\sigma}_{1}$ over the UC, we simply divided the resultant tractions $\left(\bar{R}_{i j}\right)$ on the boundary surfaces along direction-1 from the areas $\left(A_{i}\right)$ of the corresponding boundary surfaces. Using these total average values, the coefficients $\bar{S}_{11}^{E}, \bar{S}_{12}^{E}, \bar{S}_{13}^{E}$, and $\bar{d}_{31}^{E}$ can be computed using the matrix form of Eq. (3). Due to zero stresses and electric fields except along direction- 1 , the first row become $\bar{S}_{11}^{E}={ }^{1} \bar{\varepsilon}_{1} / \bar{\sigma}_{1}$. Then from second, third and ninth row, the other coefficients can be calculate as $\bar{S}_{12}^{E}={ }^{\varepsilon_{2}} / \bar{\sigma}_{1}, \bar{S}_{13}^{E}=\bar{\varepsilon}_{3} / \bar{\sigma}_{1}$, and $\bar{d}_{31}^{E}=\bar{D}_{3 / \bar{\sigma}_{1}}$. Figure 3 (c) and (d) illustrates the variation of the stiffness coefficients $\bar{C}_{11}^{E}$ and $\bar{C}_{12}^{E}$ with porosity in the 3-0 and 3-1 type piezoelectric foam. This figure shows that the elastic properties are anisotropic and exhibit similar trends for both type of foams. It is demonstrated that the piezoelectric material designed with 3-0 connectivity exhibit higher stiffnesses as compared to materials with 3-1 connectivity for the range of porosity considered. The variation shows highly nonlinear dependence on the porosity and found in reasonably good agreement with the FE results obtained using the PBCs and analytical solutions. In addition to other piezoelectric performance, the effect of porosity connectivity is important to consider for mechanical performance of piezoelectric foams. 


\section{Case 1 - Axial mechanical loading transverse to the poling direction (x-axis)}
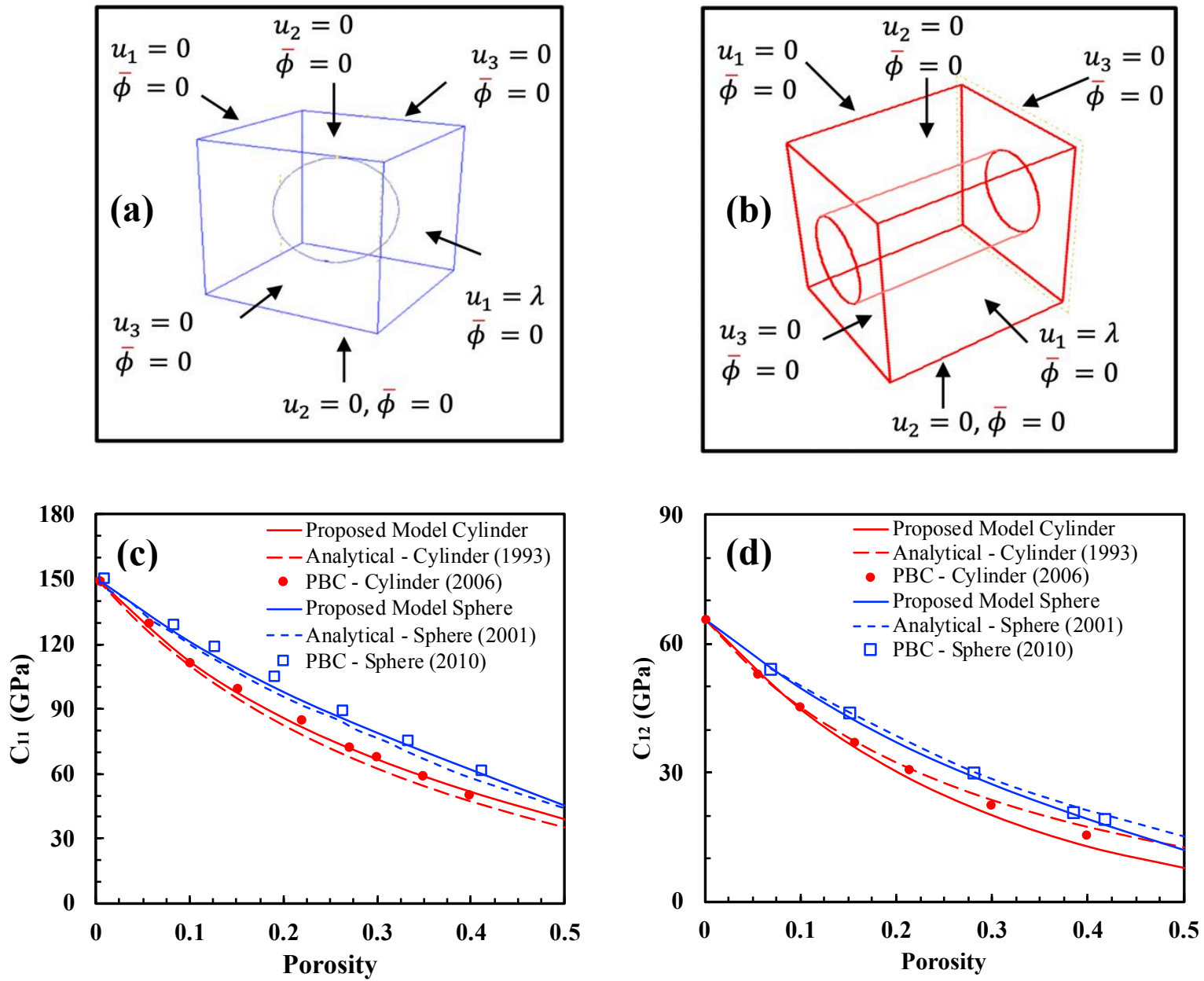

Figure 3: Boundary condition for axial mechanical loading perpendicular to poling direction for (a) 3-0 type-, and (b) (3-1) type foam. Variation in the stiffness constants with porosity in the piezoelectric foam (c) $C_{11}^{E}$ (d) $C_{12}^{E}$.

For the calculation of the effective coefficients $\bar{S}_{33}^{E}$ we prescribed a displacement in z direction on surface $\mathrm{Z}+$ as shown in Figure 4(a) and (b) such that except the stress in the direction-3 $\left(\bar{\sigma}_{3}\right)$, all other macroscopic stresses and gradients of electric potential $\left(\bar{E}_{i}\right)$ become zero. This set of boundary conditions allows us to compute the effective compliance matrix coefficients $\bar{S}_{31}^{E}, \bar{S}_{32}^{E}, \bar{S}_{33}^{E}$ 
, and $\bar{d}_{33}^{E}$. Due to zero stresses and electric fields except along direction-3, the third row become $\bar{S}_{33}^{E}$ $=\bar{\varepsilon}_{3} / \bar{\sigma}_{3}$. The variation of the $\mathrm{C}_{33}$ with porosity obtained from the proposed $\mathrm{BCs}$ and $\mathrm{PBC}$ are shown in Figure 4. Excellent agreement was found with both analytical solution and FE results obtained using the PBCs. Contrary to $\bar{C}_{11}^{E}$ and $\bar{C}_{12}^{E}$ the $\bar{C}_{33}^{E}$ results show mild non-linear reduction as a function of increase in porosity for both 3-0 and 3-1 foam. Here the 3-1 connectivity showed higher stiffness as compare to the 3-0 connectivity due to the reason that the poling direction is along the z-axis foam and 3-1 type connectivity has cylindrical (through hole) porosity parallel to the direction of the poling.

\section{Case 3 - Axial mechanical loading along to the poling direction (x-axis)}
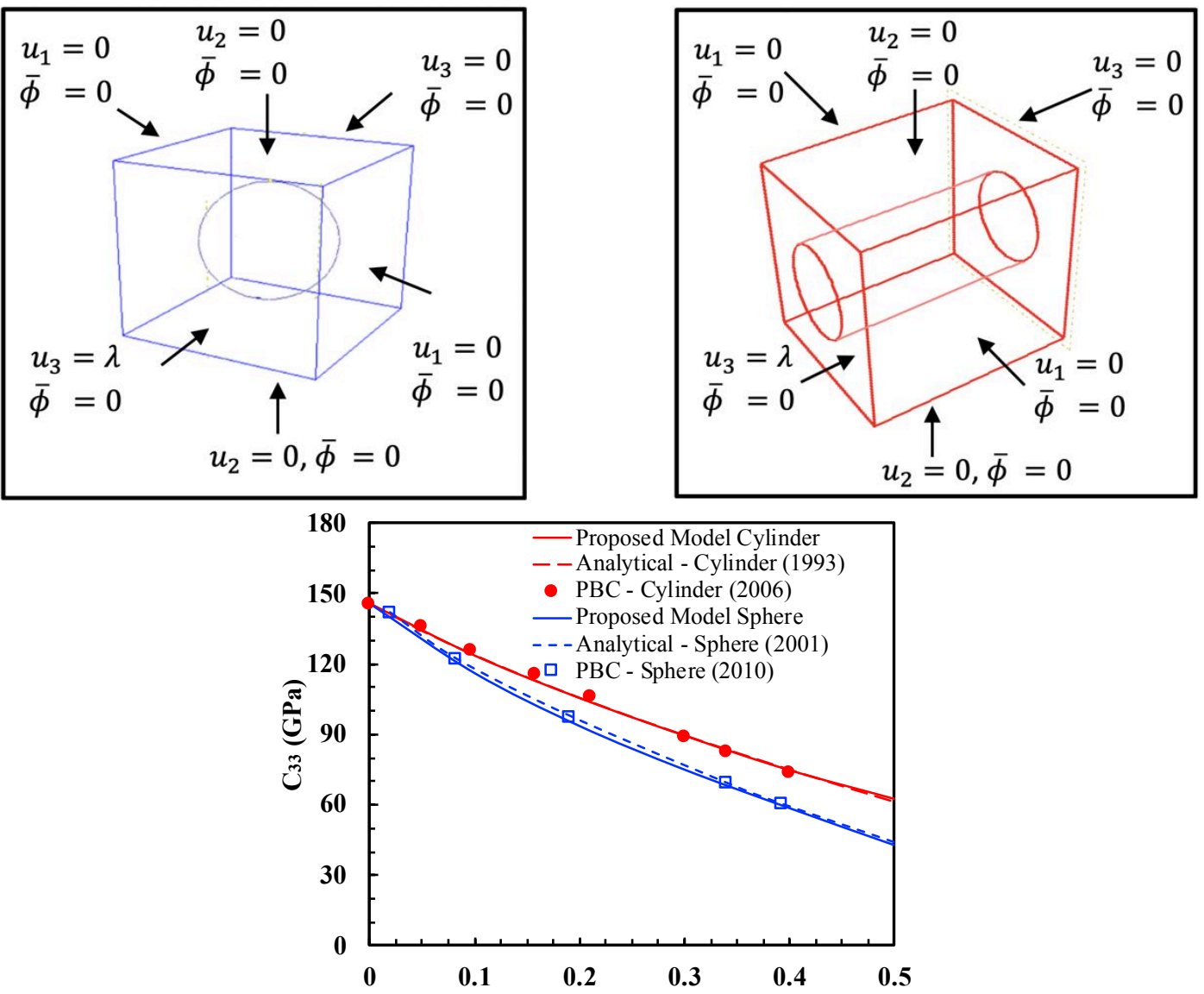
Figure 4: Boundary condition for axial mechanical loading along poling direction for (a) 3-0 type-, and (b) (31) type foam. (c) Out of plane stiffness constant $C_{33}^{E}$ variation with porosity in the piezoelectric foam.

Next, we determined the piezoelectric coupling constants. The piezoelectric strain coefficients $\bar{d}_{15}^{E}$ was determined when we prescribed an electric potential along the direction- 1 on surface $\mathrm{X}^{+}$ directions, as shown in Figure 5 (a) and (b). The variation in the shear-coupling-based piezoelectric properties with respect to porosity is shown in Figure 5 (c). Excellent agreement was found with the FE results obtained using the PBCs but reasonable agreement with the analytical solutions. In general, the piezoelectric properties of architected materials are independent of the topology of the microstructure, for example in honey comb structures (Iyer et al., 2015). However, a significant dependence on the topology is observed in the piezoelectric properties for both 3-0 and 3-1 type piezoelectric foam. It is also observed that 3-0 piezoelectric exhibit strong dependence on porosity on the piezoelectric sensitivity to shear-type piezoelectric properties as compared to 3-1 connectivity.
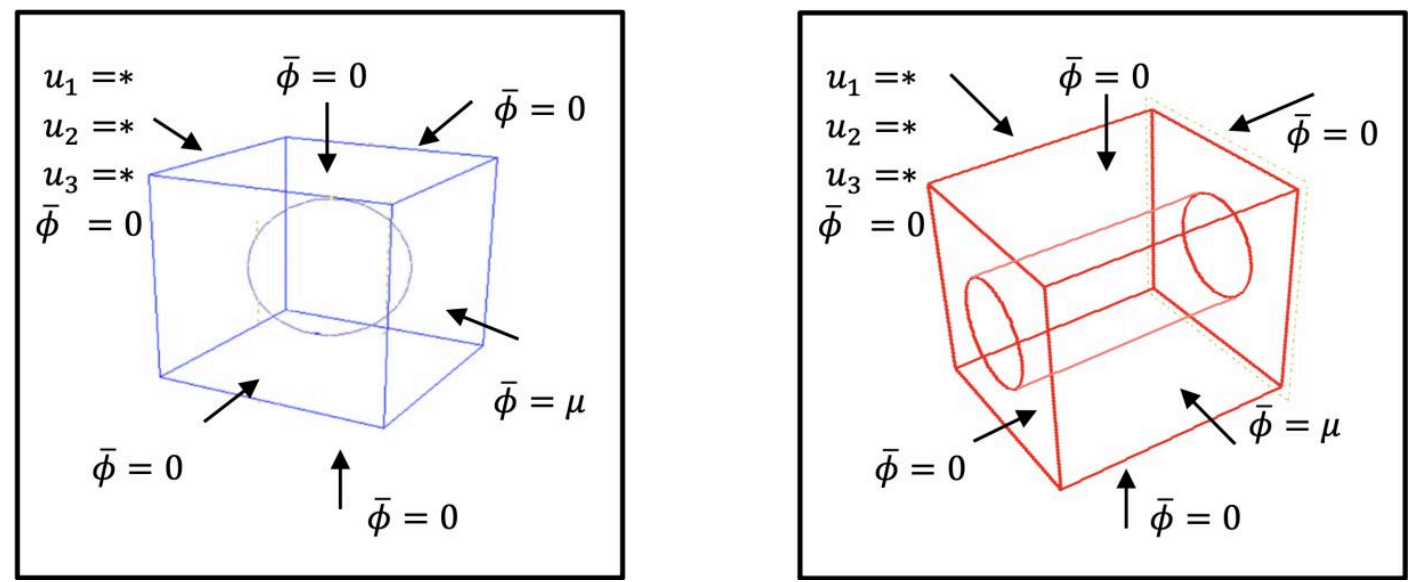


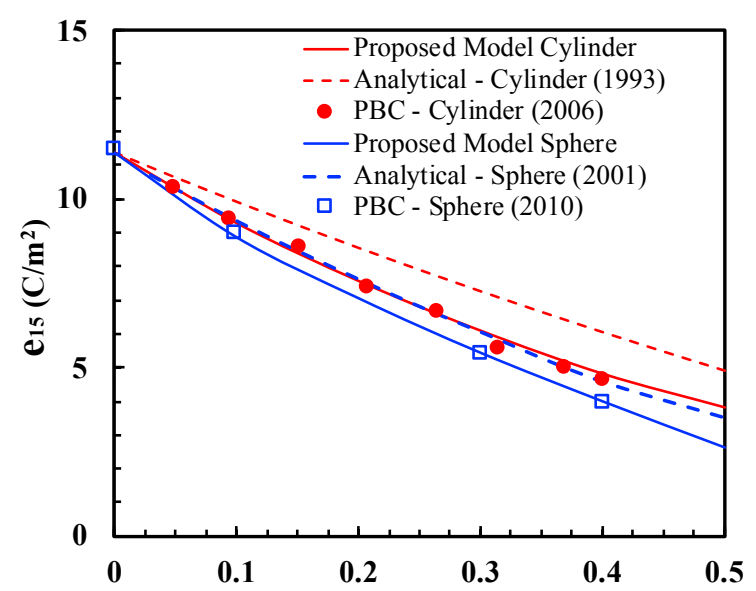

Figure 5: Variation in the overall piezoelectric constants with porosity in the 3-0 type piezoelectric foam.

Next we compared the principal normal piezoelectric $\bar{d}_{33}^{\sigma}$, and dielectric $\bar{\kappa}_{33}^{\sigma}$ constants, which were obtained by prescribing an electric potential along the direction- 3 on surface $\mathrm{Z}+$ as shown in Figure 6 (a) and (b). The variation of principal normal piezoelectric constants with respect to porosity is shown in Figure 6 (c). A significant dependence on the topology is observed in the piezoelectric properties for both 3-0 and 3-1 type piezoelectric foam, but the normal-based piezoelectric properties show almost linear dependence on the porosity. Figure 6 (d) showed the effective dielectric properties under constant stress as a function of porosity for 3-0 and 3-1 type foam. The dielectric constants exhibit linear dependence and their values are decreased with an increase in the porosity. However similar to longitudinal stiffness constants $\mathrm{C}_{33}$, the longitudinal piezoelectric and dielectric constants with 3-1 connectivity exhibit higher performance as compared to materials with 3-0 connectivity for the range of porosity considered. Results obtained from the proposed $\mathrm{BCs}$ are in excellent agreement with the results those obtained from FE results with PBCs and analytical solutions. 
Case 9 - Potential gradient along the poling direction (z-axis)
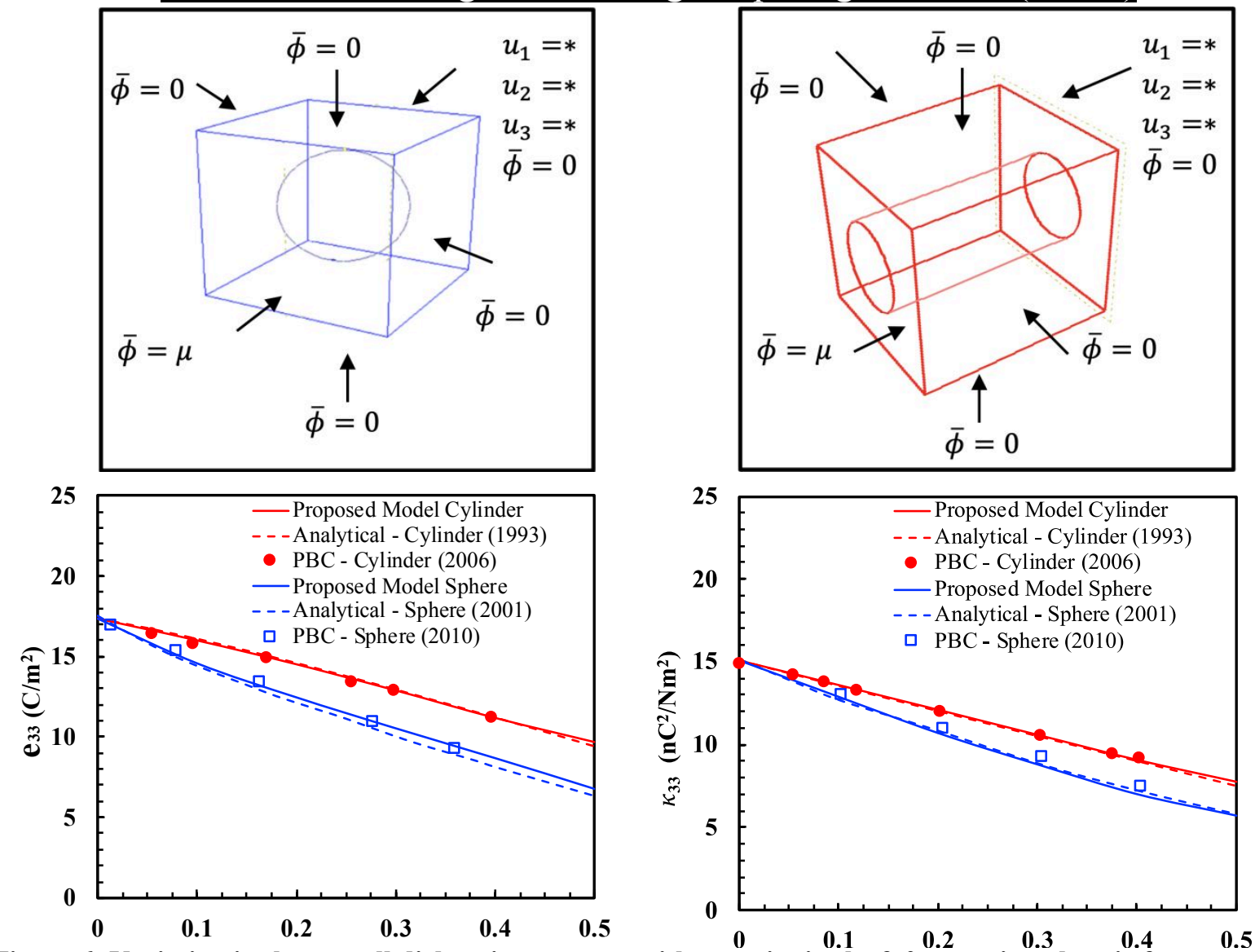

Figure 6: Variation in the overall dielectric constants with porosity in the 3-0 type piezoelectric foam.

\section{Effective Figure of Merit}

Porous piezoelectric materials are of interest in various industrial applications, such as hydrophones, ultrasound imager, and their performance in these applications are evaluated based on effective figure of merit. For hydrophones applications, the figure of merits that have direct relevance are hydrostatic strain coefficient $\left(d_{h}=d_{31}+d_{32}+d_{33}\right)$, hydrostatic figure of merit $d_{h} g_{h}$ with $g_{h}=g_{31}+g_{32}+g_{33}$, acoustic impedance $Z=\left[\rho \mathrm{C}_{33}^{E}\right]^{1 / 2}$, electromechanical thickness mode coupling factor $k_{t}=\left[1-\mathrm{C}_{33}^{E} / \mathrm{C}_{33}^{D}\right]^{1 / 2}=e_{33} /\left[\kappa_{33}^{\varepsilon} \mathrm{C}_{33}^{D}\right]^{1 / 2}$ (Kar-Gupta and Venkatesh, 2006).

Figure 7 presents the variation of the selected figures of merit with increasing porosity for the 3-0 and 3-1 type piezoelectric foam. All the FOMs have shown an enhanced response for 3-0 type 
piezoelectric foam and showed strong dependence on the porosity. All the figure of merits results obtained from the proposed approach are in excellent agreement with the ones those obtained from FE results obtained using the PBCs as shown in Figure 7.

The hydrostatic strain coefficient $d_{h}$ is almost independent of the porosity for 3-1 foam, while the hydrostatic strain coefficient $d_{h}$ shows a linear variation of porosity for 3-0 foam and increased with the increase of the porosity as shown in Figure 7 (a). Next we analyzed the hydrostatic figure of merit $d_{h} g_{h}$. For 3-1 foam the hydrostatic strain coefficient shows a very mild effect on the variation of porosity and shows linear dependence with an increase slightly with the increase of porosity. However, 3-0 foam shows nonlinear dependence on the porosity and increase significantly with an increase in the porosity as shown in Figure 7 (b). The large enhancement in the $d_{h} g_{h}$ is obtained due to the inverse relationship between $g_{33}$ and $\kappa_{33}$. Therefore, an increase of porosity can cause a decrease in the dielectric constant results an increase in $g_{h}$.

The acoustic impedance $\mathrm{Z}$ generally decreases with an increase in the porosity. Figure 7 (c) shows that for both 3-0 type and 3-1 type piezoelectric foam the $\mathrm{Z}$ follows linear relation with the increase of the porosity. These computational studies are agreed well with the experimental observations (Bast and Wersing, 1989) and have shown a similar trend in the $\mathrm{Z}$ values with an increase of porosity and confirmed that the 3-0 foam strongly dependent on the porosity as compare to 3-1 foam. Figure 7 (d) shows the electromechanical coupling constant for both 3-0 and 3-1 type foam. Both foams show an increase in coupling constant values with an increase in the porosity but 3-1 foam shows little stronger dependence on the porosity as compared to 3-0 foam.

In comparison to their bulk constituent, both 3-0 and 3-1 piezoelectric foam exhibit an enhanced response. The 3-0 foam exhibit significant increase in their hydrostatic figure of merit. These enhancements are coupled with decrease in the acoustic impedance values. The hydrostatic figure 
of merit and acoustic impedance are the two most important transducer parameters for the design of hydrophones. From the point of view of transducer design, the Z- $d_{h} g_{h}$ relation is very important. With the increase of porosity, an increase in the $d_{h} g_{h}$ are accompanied by the decreases in acoustic impedance. This combination of increased sensitivity-based figures of merit and reduced acoustic impedance is unique, as it is impossible to realize using piezo-composites or bulk PMs (M. L. Dunn and Taya, 1993). Using topology optimization, Sigmund et al (Sigmund et al., 1998) optimized porous microstructure design and showed enhanced performance of $d_{h}$ and $d_{h} g_{h}$ over pure piezoceramics. Thus, it is possible to design piezoelectric with controlled porosity with the desirable electro-mechanical characteristics.
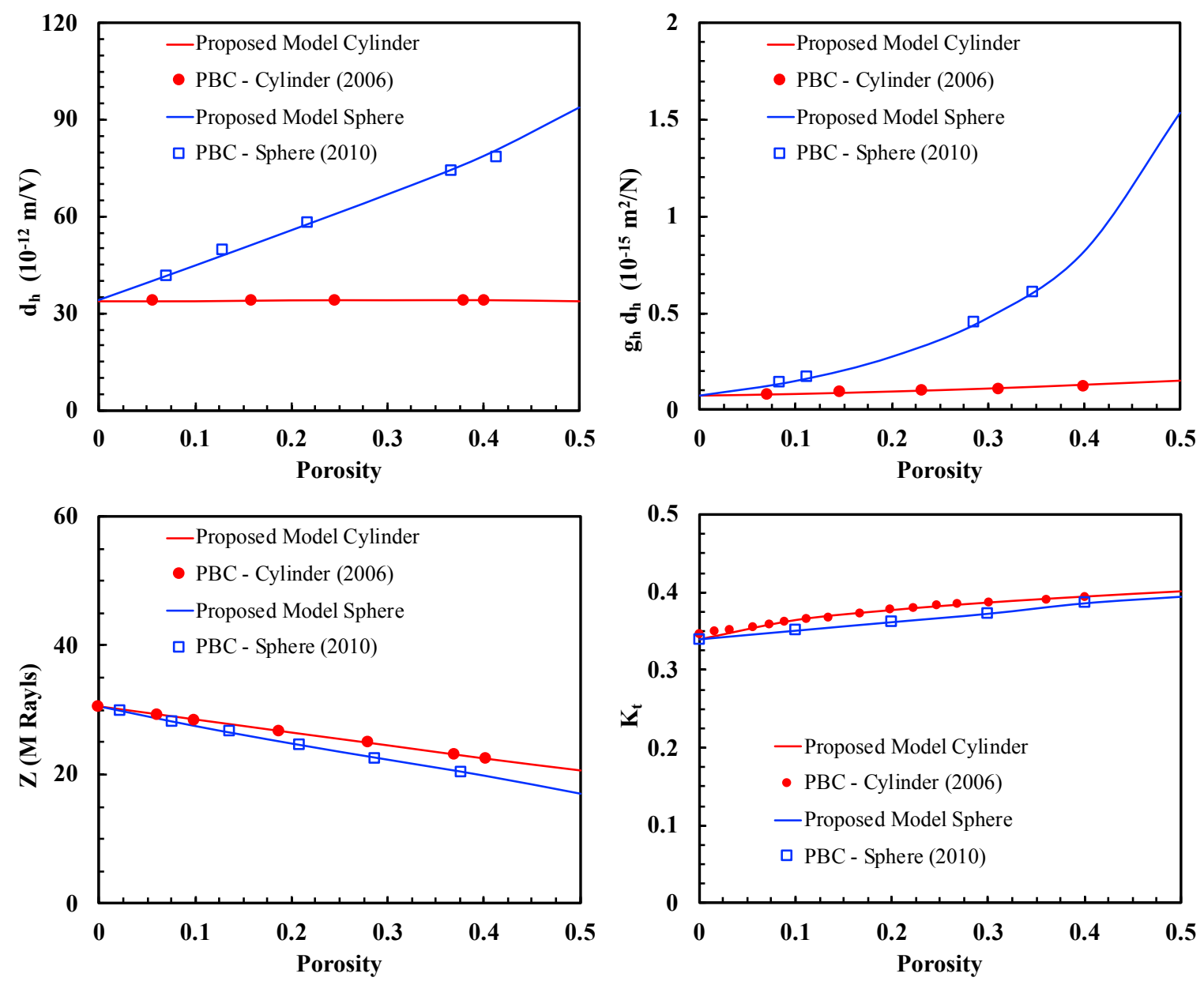
Figure 7: Variation in figures of merit with porosity in the 3-0 type piezoelectric foam. (a) hydrostatic strain coefficient, (b) hydrostatic figure of merit, (c) acoustic impedance (d) electromechanical coupling constant.

The FE results show that the mechanical and piezoelectric properties of the piezoelectric materials can be optimized by tailoring the porosity of 3-0 type piezoelectric foam and thus a unique combination of tunable properties can be realized as required in various practical applications. The interplay between architecture connectivity and material anisotropy leads to a wide range of architecture-dependent elastic, piezoelectric and dielectric properties that differ substantially from the properties of the architecture base material. The FE results have confirmed excellent electromechanical properties of the 3-0 piezoelectric foam. The presented approach showed that novel design of piezoelectric foam with controlled architected porosity can be designed that are desirable in unique devices for sensing or actuating applications.

\section{Conclusions}

A micromechanical-finite element homogenization approach is presented to compute the electromechanical properties of the architected periodic piezoelectric foam with controlled porosity of spherical and cylindrical shape, with 3-0 and 3-1 type connectivity, respectively. A set of mixed boundary conditions equivalent to a periodic boundary conditions for architected periodic piezoelectric foam with certain symmetries are used to ensure that the displacements and the electric potential of a UC are compatible across its boundaries with that of the adjacent UCs. Results show that the 3-0 type piezoelectric foam exhibits a unique combination of properties which cannot be realized by bulk piezoelectric materials and 3-0 type foam offer moreover enhanced hydrostatic figure of merit as compared to piezoelectric foams with 3-1 connectivity, and more suitable to hydrophone applications. The variation of elastic, piezoelectric and dielectric 
properties of 3-0 and 3-1 foams with porosity were computed, and compared with the analytical solutions of both 3-0 and 3-1 foams that were obtained using micromechanics theory, and finiteelement homogenization results that were obtained by employing the periodic boundary conditions. Excellent agreement between the proposed modeling approach and the ones obtained using analytical, and FE results available in the literature showed that the proposed modeling approach is simpler and flexible for the calculation of the effective electro-mechanical properties of architected piezoelectric foam with tunable properties while exhibiting elastic anisotropy and piezoelectric activity.

\section{ACKNOWLEDEMENTS}

This publication is based on work supported by the Khalifa University of Science and Technology under Award No. CIRA-2018-15 and Abu Dhabi Award for Research Excellence (AARE-2019) under project number 8434000349 .

\section{REFERENCES}

Banno H (1985) Theoretical equations for dielectric and piezoelectric properties of ferroelectric composites based on modified cubes model. Japanese Journal of Applied Physics 24(S2): 445.

Bast U and Wersing W (1989) The influence of internal voids with 3-1 connectivity on the properties of piezoelectric ceramics prepared by a new planar process. Ferroelectrics 94(1): 229-242.

Berger H, Kari S, Gabbert U, et al. (2006) Unit cell models of piezoelectric fiber composites for numerical and analytical calculation of effective properties. Smart Materials and Structures 15(2): 451.

Bowen CR and Topolov VY (2003) Piezoelectric sensitivity of PbTiO 3-based ceramic/polymer composites with 03 and 3-3 connectivity. Acta materialia 51(17): 4965-4976.

Choudhry RS, Khan KA, Khan SZ, et al. (2016) Micromechanical modeling of 8-harness satin weave glass fiberreinforced composites. Journal of Composite Materials: 0021998316649782. DOI: $10.1177 / 0021998316649782$.

Dunn ML (1994) Electroelastic Green's functions for transversely isotropic piezoelectric media and their application to the solution of inclusion and inhomogeneity problems. International Journal of Engineering Science 32(1): 119-131. DOI: 10.1016/0020-7225(94)90154-6.

Dunn Martin L. and Taya M (1993) Electromechanical Properties of Porous Piezoelectric Ceramics. Journal of the American Ceramic Society 76(7): 1697-1706. DOI: 10.1111/j.1151-2916.1993.tb06637.x. 
Dunn M. L. and Taya M (1993) Micromechanics predictions of the effective electroelastic moduli of piezoelectric composites. International Journal of Solids and Structures 30(2): 161-175.

Geis S, Löbmann P, Seifert S, et al. (2000) Dielectric properties of PZT aerogels. Ferroelectrics 241(1): 75-82.

Getman I and Lopatin S (1996) Theoretical and experimental investigation of porous PZT ceramics. Ferroelectrics 186(1): 301-304. DOI: 10.1080/00150199608218088.

Haun MJ and Newnham RE (1986) An experimental and theoretical study of 1-3 AND 1-3-0 piezoelectric PZTPolymer composites for hydrophone applications. Ferroelectrics 68(1): 123-139.

Hikita K, Yamada K, Nishioka M, et al. (1983) Piezoelectric properties of the porous PZT and the porous PZT composite with silicone rubber. Ferroelectrics 49(1): 265-272. DOI: 10.1080/00150198308244698.

Iyer S and Venkatesh TA (2010) Electromechanical response of porous piezoelectric materials: Effects of porosity connectivity. Applied Physics Letters 97(7): 072904.

Iyer S and Venkatesh TA (2011) Electromechanical response of (3-0) porous piezoelectric materials: Effects of porosity shape. Journal of Applied Physics 110(3): 034109.

Iyer S, Alkhader M and Venkatesh TA (2015) Electromechanical behavior of auxetic piezoelectric cellular solids. Scripta Materialia 99: 65-68.

Jiang M, Jasiuk I and Ostoja-Starzewski M (2002) Apparent thermal conductivity of periodic two-dimensional composites. Computational Materials Science 25(3): 329-338.

Kanit T, Forest S, Galliet I, et al. (2003) Determination of the size of the representative volume element for random composites: statistical and numerical approach. International Journal of solids and structures 40(13): 36473679 .

Kara H, Ramesh R, Stevens R, et al. (2003) Porous PZT ceramics for receiving transducers. Ultrasonics, Ferroelectrics, and Frequency Control, IEEE Transactions on 50(3): 289-296.

Kar-Gupta R and Venkatesh TA (2006) Electromechanical response of porous piezoelectric materials. Acta Materialia 54(15): 4063-4078.

Kar-Gupta R and Venkatesh TA (2008) Electromechanical response of piezoelectric composites: Effects of geometric connectivity and grain size. Acta Materialia 56(15): 3810-3823.

Khan KA and Abu Al-Rub RK (2017) Time dependent response of architectured Neovius foams. International Journal of Mechanical Sciences 126: 106-119. DOI: 10.1016/j.ijmecsci.2017.03.017.

Khan KA and Khan MA (2019) 3-3 piezoelectric metamaterial with negative and zero Poisson's ratio for hydrophones applications. Materials Research Bulletin 112: 194-204. DOI: 10.1016/j.materresbull.2018.12.016.

Khan KA and Muliana AH (2009) A multi-scale model for coupled heat conduction and deformations of viscoelastic functionally graded materials. Composites Part B: Engineering 40(6). Blast/impact on engineered (nano)composite materials: 511-521. DOI: 10.1016/j.compositesb.2009.02.003.

Khan KA, Al-Mansoor S, Khan SZ, et al. (2019) Piezoelectric Metamaterial with Negative and Zero Poisson's Ratios. Journal of Engineering Mechanics 145(12). American Society of Civil Engineers: 04019101. DOI: 10.1061/(ASCE)EM.1943-7889.0001674. 
Lee S-H, Jun S-H, Kim H-E, et al. (2007) Fabrication of Porous PZT-PZN Piezoelectric Ceramics With High Hydrostatic Figure of Merits Using Camphene-Based Freeze Casting. Journal of the American Ceramic Society 90(9): 2807-2813. DOI: 10.1111/j.1551-2916.2007.01834.x.

Li S (2008) Boundary conditions for unit cells from periodic microstructures and their implications. Composites Science and Technology 68(9): 1962-1974.

Marselli S, Pavia V, Galassi C, et al. (1999) Porous piezoelectric ceramic hydrophone. The Journal of the Acoustical Society of America 106(2): 733. DOI: 10.1121/1.427091.

Martínez-Ayuso G, Friswell MI, Adhikari S, et al. (2017) Homogenization of porous piezoelectric materials. International Journal of Solids and Structures 113-114: 218-229. DOI: 10.1016/j.jjsolstr.2017.03.003.

Mikata Y (2001) Explicit determination of piezoelectric Eshelby tensors for a spheroidal inclusion. International Journal of Solids and Structures 38(40-41): 7045-7063. DOI: 10.1016/S0020-7683(00)00419-4.

Mori $\mathrm{T}$ and Tanaka K (1973) Average stress in matrix and average elastic energy of materials with misfitting inclusions. Acta Metallurgica. DOI: 10.1016/0001-6160(73)90064-3.

Nagata K, Igarashi $\mathrm{H}$, Okazaki K, et al. (1980) Properties of an Interconnected Porous $\mathrm{Pb}(\mathrm{Zr}$, $\mathrm{Ti}) \mathrm{O}_{3}$ Ceramic. Japanese Journal of Applied Physics 19(1): L37-L40. DOI: 10.1143/JJAP.19.L37.

Newnham RE, Skinner DP and Cross LE (1978) Connectivity and piezoelectric-pyroelectric composites. Materials Research Bulletin 13(5): 525-536. DOI: 10.1016/0025-5408(78)90161-7.

Padurariu L, Curecheriu L, Galassi C, et al. (2012) Tailoring non-linear dielectric properties by local field engineering in anisotropic porous ferroelectric structures. Applied Physics Letters 100(25): 252905. DOI: 10.1063/1.4729878.

Roscow J, Zhang Y, Taylor J, et al. (2015) Porous ferroelectrics for energy harvesting applications. The European Physical Journal Special Topics 224(14): 2949-2966. DOI: 10.1140/epjst/e2015-02600-y.

Roscow JI, Lewis RWC, Taylor J, et al. (2017) Modelling and fabrication of porous sandwich layer barium titanate with improved piezoelectric energy harvesting figures of merit. Acta Materialia 128: 207-217. DOI: 10.1016/j.actamat.2017.02.029.

Roscow JI, Zhang Y, Kraśny MJ, et al. (2018) Freeze cast porous barium titanate for enhanced piezoelectric energy harvesting. Journal of Physics D: Applied Physics 51(22). IOP Publishing: 225301. DOI: 10.1088/13616463/aabc81.

Sigmund O, Torquato S and Aksay IA (1998) On the design of 1-3 piezocomposites using topology optimization. Journal of materials research 13(4): 1038-1048.

Silva ECN, Fonseca JSO and Kikuchi N (1998) Optimal design of periodic piezocomposites. Computer Methods in Applied Mechanics and Engineering 159(1-2): 49-77.

Silva EN, Fonseca JO and Kikuchi N (1997) Optimal design of piezoelectric microstructures. Computational mechanics 19(5): 397-410.

Topolov VY and Bowen CR (2008) Electromechanical Properties in Composites Based on Ferroelectrics. Springer Science \& Business Media.

Ueda J, Secord TW and Asada HH (2010) Large effective-strain piezoelectric actuators using nested cellular architecture with exponential strain amplification mechanisms. IEEE/ASME Transactions on Mechatronics 15(5): 770-782. 
Wadley HN (2006) Multifunctional periodic cellular metals. Philosophical Transactions of the Royal Society of London A: Mathematical, Physical and Engineering Sciences 364(1838): 31-68.

Xia Z, Zhang Y and Ellyin F (2003) A unified periodical boundary conditions for representative volume elements of composites and applications. International Journal of Solids and Structures 40(8): 1907-1921. DOI: 10.1016/S0020-7683(03)00024-6.

Yang J (2006) An Introduction to the Theory of Piezoelectricity. Springer Science \& Business Media.

Zhang Y, Roscow J, Lewis R, et al. (2018) Understanding the effect of porosity on the polarisation-field response of ferroelectric materials. Acta Materialia 154: 100-112. 
2021-01-05

\section{Micromechanical modeling of}

architected piezoelectric foam with

simplified boundary conditions for

hydrophone applications

Khan, Kamran Ahmed

SAGE

Khan KA, Alarafati HK, Khan MA. (2021) Micromechanical modeling of architected piezoelectric foam with simplified boundary conditions for hydrophone applications. Journal of Intelligent Material Systems and Structures, Volume 32, Issue 15, September 2021, pp. 1700-1712 https://doi.org/10.1177/1045389X20983912

Downloaded from Cranfield Library Services E-Repository 